\title{
Whole Body Interaction Using the Grounded Bar Interface
}

\author{
Bong-gyu Jang, Hyunseok Yang, and Gerard J. Kim
}

\author{
Digital Experience Laboratory \\ Korea University, Seoul, Korea \\ gjkim@korea.ac.kr
}

\begin{abstract}
Whole body interaction is an important element in promoting the level of presence and immersion in virtual reality systems. In this paper, we investigate the effect of "grounding" the interaction device to take advantage of the significant passive reaction force feedback sensed throughout the body, and thus in effect realizing the whole body interaction without complicated sensing and feedback apparatus. An experiment was conducted to assess the task performance and level of presence/immersion, as compared to a keyboard input method, using a maze navigation task. The results showed that while the G-Bar did induce significantly higher presence and the task performance (maze completion time and number of wall collisions) was on par with the already familiar keyboard interface. The keyboard user instead had to adjust and learn how to navigate faster and not collide with the wall over time, indicating that the whole body interaction contributed to a better perception of the immediate space. Thus considering the learning rate and the relative unfamiliarity of GBar, with sufficient training, G-Bar could accomplish both high presence/immersion and task performance for $\mathrm{s}$.
\end{abstract}

Keywords: Whole-body interaction, Presence, Immersion, Task performance, Isometric interaction.

\section{Introduction}

One of the defining characteristics of virtual reality is the provision of "presence" [1], the feeling of being contained in the content. Many researches have identified the elements that contribute to enhance the level of presence [1][8], and one such element is the use of "whole body" interaction whose strategy is to leverage on as many sensory and motor organs as possible [2].

In this paper, we introduce an interface called the "G-Bar" (Grounded Bar), a twohanded isometric device that is fixed to the ground (grounded) for a variety of interactive tasks including navigation, and object selection and manipulation. The interface is "whole body" because it is basically operated with two hands, and since it is grounded, it also indirectly involves the interactions through the legs and body parts in between (see Figure 1). Since the user also needs to move one's head/neck in order to view and scan the environment visually, virtually all parts of the body become active. Moreover, since the device is isometric and senses the user's pressure input, 
the user can express dynamic interactions more naturally [3][4][5]. In addition, we formally evaluate and validate the projected merits of the whole body interaction induced by the "grounded" device such as G-Bar.

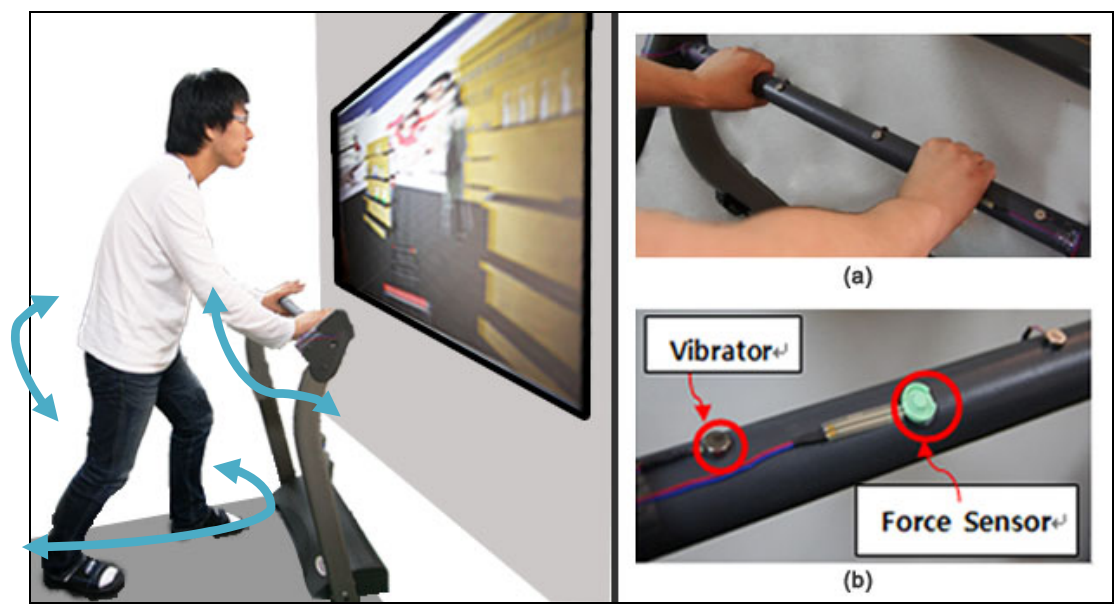

Fig. 1. The G-Bar in usage for navigating and selecting objects in a virtual environment. The reaction force resulting from the two-hand interaction with the grounded device propagates throughout the body (right). More detailed view of the G-Bar prototype (left).

\section{Related Work}

Employing whole body interaction is an effective method to enhance the immersive quality of interactive contents [6][7][8]. However, whole body interaction does not necessarily require separate sensing and feedback mechanisms for the body parts involved. Through clever interaction and interface design, whole body interaction can be induced through gesture latitude and minimal sensing. For instance, the arcade game "Dance Dance Revolution" [9] utilizes a very simple foot switch pad, but the interaction is designed to induce the use of whole body (similarly for Nintendo Wii based games [10]).

This concept is somewhat related to that of the "passive" haptics, an inexpensive and creative way to use natural reaction force feedback (e.g. tangible props). However, props are usually light weight and fragile, limiting the users in applying high amount of forces. Meehan et al. [11] demonstrated the utility of passive haptics with grounded prop (a ledge) which has significantly enhanced the level of presence for their virtual cliff environment. A large reaction force is more likely to propagate throughout and stimulate the whole the body.

Isometric input is also known to increase interaction realism by allowing dynamic expression through the input [3][4][5]. G-Bar combines all these aforementioned elements in hopes to creating effective interaction and compelling user experience.

On the other hand, the effect of whole body toward the task performance is unclear. For one, the relationship between presence/immersion and task performance is generally viewed as being task dependent [1][8]. 


\section{G-Bar}

G-Bar is implemented by installing low cost pressure sensors and vibrating motors on a bar handle. Four pressure sensors (two at each end of the bar) realize the isometric input and the four vibrating motors (laid out in a regular interval along the bar) give directional feedback cues in addition to the natural reaction force.

Thus G-Bar is particularly appropriate for tasks that involve frequent and dynamic contact with the environment or interaction object. A typical example might be navigating and passing through a bumping crowd, riding and directly controlling a vehicle such as a cart, motor cycle or hang-glider. In fact, the "bar" resembles the control handles used for some of these vehicles (e.g. motor cycle handle), and such metaphors can be even more helpful.

While object selection and manipulation might not really involve whole body interaction in the real life, by exaggerating the extent of the required body parts involved, we posit that it could be a one way to maximize the virtual experience. Figure 2 shows how one might be able to navigate through the virtual space by combinations of simple two handed isometric push (forward) and pull (backward) actions. In the selection mode, the same interaction technique can be used for controlling the virtual ray/cone, then applying the grasping action for final selection (right hand grasp) and undo (left hand grasp). Once the object is selected, it can be rotated and moved in a similar fashion as well. Despite the seemingly natural interaction metaphors, the sheer unfamiliarity will require the users some amount of learning.

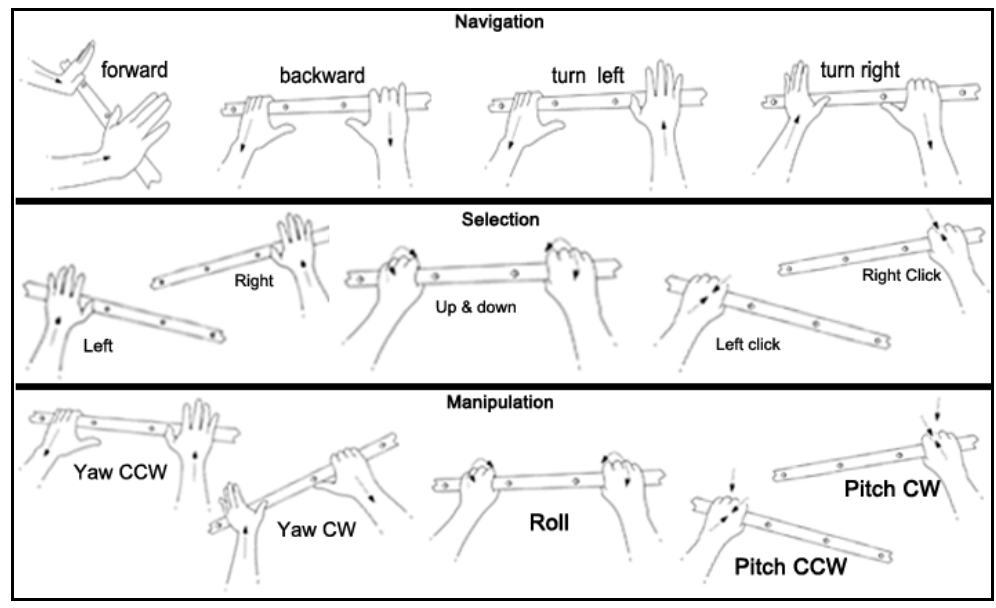

Fig. 2. Navigation, and object selection and manipulation through combinations of push, pull, twist and grab actions with the G-Bar

\section{Experiment}

To assess the effectiveness of the proposed interaction technique, we have carried out an experiment comparing the G-Bar interface to a non-grounded interface, namely a 
keyboard input. The user was asked to navigate a fixed path in a virtual maze with a cart (in first person viewpoint) and the task performance, level of presence/immersion and general usability were measured. Our hypothesis was that the use of G-Bar would result in significantly enhanced user experience (e.g. high presence and immersion), but may not produce good task performance nor high usability without sufficient training.

\subsection{Experiment Design}

The experiment was designed as a one factor (two level) repeated measure (within subject), the sole factor being the type of the interface employed (G-Bar vs. keyboard). The subject was asked to navigate a fixed path in a maze like environment using the two interfaces (presented in a balanced order). The shown in Figure 3, the virtual maze was composed of brick walls with the directional path marked for user convenience. The subject was asked to follow and navigate the path as fast as possible but without colliding with walls as much as possible. The width of the path was set properly so that the task was not too easy, especially in making turns (after an initial pilot test). The test environment was staged as the user pushing cart (seen in the first person viewpoint) with a large box in it occluding the front end so that the user had to get the "feel" for the extent of the cart. This "feel" would be important in avoiding collision with the walls and also a quality that was thought to be better acquired with a whole body interaction and thus would produce higher task performance (at least eventually).
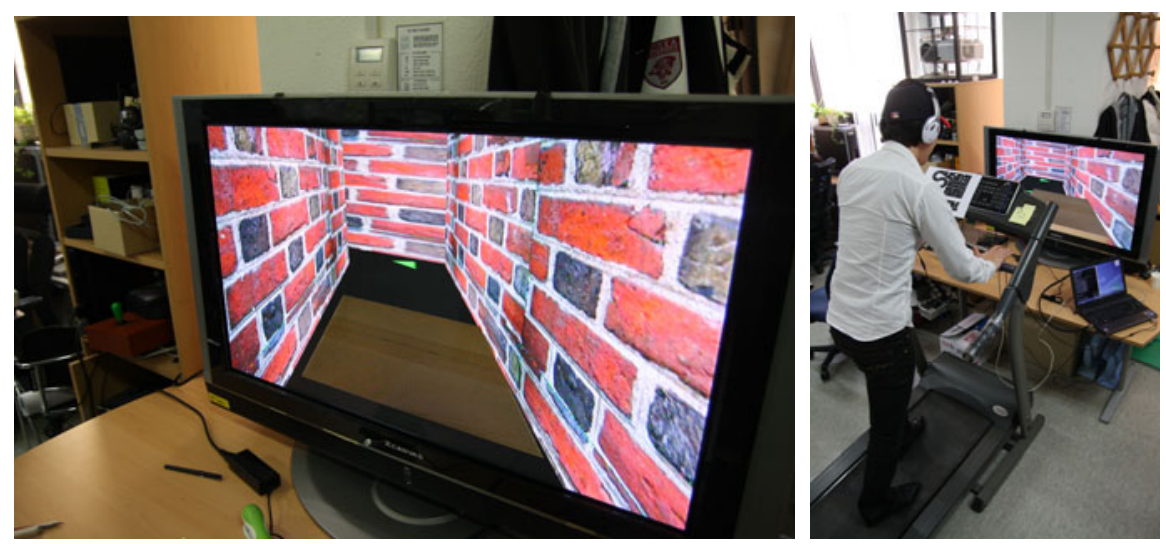

Fig. 3. A snapshot of the virtual maze used in the comparative experiment (left). A subject navigating the virtual maze using the interface set up. The G-Bar is installed on a heavy treadmill for grounding. The keyboard interface was also placed on the same place where the G-Bar was installed (right).

The measured dependent variables were the task completion time, accuracy (e.g. no. of collision), subjective presence/immersion score (collected through a survey) and other general usability (we omit the content of the survey for lack of space). 


\subsection{Experiment Process}

A total of 16 subjects participated in the experiment (13 males / 3 Females). Average age of the subject was 27.18 and mostly college undergraduate or graduate students recruited on campus, all with previous experiences in keyboard/mouse based computer interface and using supermarket carts. They were given proper compensation for the participation.

The subject was first given some amount of training until one got sufficiently familiarized with the G-Bar. However, due to the prior familiarity with the keyboard interface, the competency of G-Bar usage did not match that of the keyboard. Then, the subject navigated the virtual maze using the two interfaces presented in a balanced order. The subject tried out the maze three times over which the same maze was used to measure the learning effect of the interface itself. The learning effect (of the maze) or bias due to using the same maze over the trials or over the different treatments was deemed minimal because the task was simply to follow the marked path (rather than finding the opening path).

Since G-Bar is a grounded interface, it was attached to a front horizontal bar on a (heavy) treadmill (see Figure 3, the moving walk of treadmill in the bottom was not used). To set the environment condition equally, when the keyboard was used, it was placed on the same position as the G-Bar.

The quantitative dependent variables were captured automatically through the test program software and the presence/immersion and usability survey was taken after the subject carried out both treatments.

\section{Experiment Results}

Figure 4 shows the experiment results with the task performance (task completion time and no. of wall collisions) over three trials. Contrary to our expectation, despite the relative familiarity of the keyboard interface, the users performed generally better with the G-Bar interface (even though statistically significant differences were not observed). Moreover, the keyboard interface showed more learning effect than the G-Bar. Therefore, we can conclude that the G-Bar (or whole body interaction) was a more proper interface (e.g. better depth/spatial perception) to begin with and in reverse forced the keyboard users to adapt and learn how not to collide or navigate better.

Figure 5 shows the experiment results with the qualitative survey responses (all measured in the 7 Likert scale). It is very interesting that in relation to the results with the task performance, the users still felt the keyboard interface was much easier. The extent of perceived level of whole body usage, force feedback (note that there was not explicit force feedback) and immersion (presence) was much higher with the G-Bar (with statistical significance). Again, we posit that such factors affected the depth and spatial perception contributing to the relatively high task performance despite the user was not totally trained to use the G-Bar. 

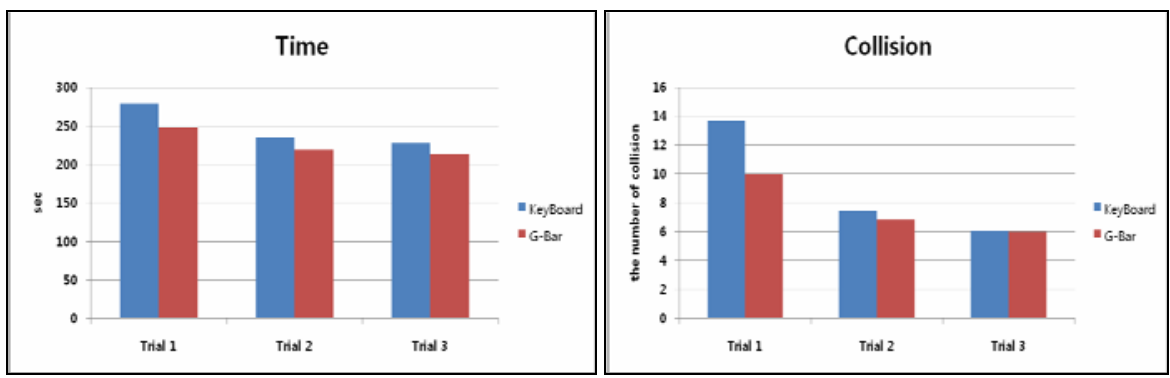

Fig. 4. Experiment results (time of completion and no. of collisions between the keyboard and G-Bar)
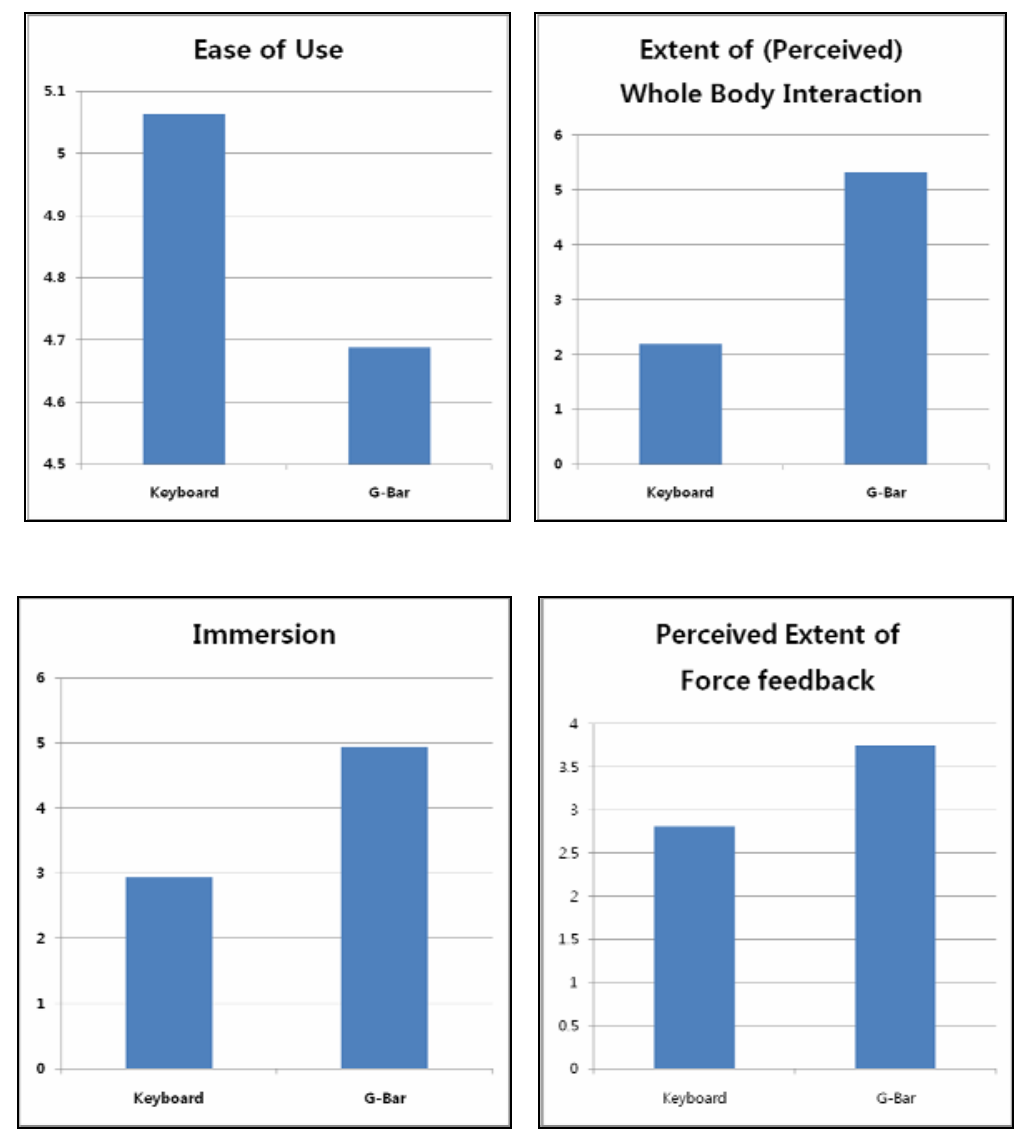

Fig. 5. Experiment results (survey questions: ease of use, extent of whole body interaction, the level of immersion, and extent of the perceived force feedback) 


\section{Conclusion}

In this paper, we presented the G-Bar, a low cost, two handed isometric whole body interface for interacting in the virtual space. The use of two hands in combination with the passive reactive feedback proved to be a contributing factor to enhanced presence. In addition, despite the novelty of the technique, after minimal training, the users were able to achieve the level of task performance comparable to the nominal non-grounded device as well. While G-Bar may not be appropriate for all types of virtual tasks (e.g. for interacting with fast moving light objects with relatively little reaction force, or for tasks that are more natural with one hand), the study shows the effectiveness of grounding the interaction device and leveraging on the naturally induced whole body experience. We believe that in combination with multimodal feedback (e.g. vibration feedback and visual simulation), the virtual experience can be further enriched.

Acknowledgements. This research was supported in part by the Strategic Technology Lab. Program (Multimodal Entertainment Platform area) and the Core Industrial Tech. Development Program (Digital Textile based Around Body Computing area) of the Korea Ministry of Knowledge Economy (MKE).

\section{References}

1. Kim, G.J.: Designing Virtual Reality Systems: A Structured Approach. Springer, Heidelberg (2005)

2. Buxton, W.: There's More to Interaction than Meets the Eye: Some Issues in Manual Input. In: Norman, D.A., Draper, S.W. (eds.) User Centered System Design: New Perspectives on Human-Computer Interaction, pp. 319-337. Lawrence Erlbaum Associates, Mahwah (1986)

3. Lecuyer, A., Coquillart, S., Kheddar, A.: Pseudo-Haptic Feedback: Can Isometric Input Devices Simulate Force Feedback. In: Proceedings of the IEEE Virtual Reality Conference, pp. 83-89 (2000)

4. Zhai, S.: Investigation of Feel for 6DOF inputs: Isometric and Elastic rate control for manipulation in 3D environments. In: Proceedings of the Human Factors and Ergonomics Society (1993)

5. Zhai, S.: User Performance in Relation to 3D Input Device Design. Computer Graphics 32(4) (1998)

6. Boulic, R., Maupu, D., Peinado, M., Raunhardt, D.: Spatial Awareness in Full-Body Immersive Interactions: Where Do We Stand? In: Boulic, R., Chrysanthou, Y., Komura, T. (eds.) MIG 2010. LNCS, vol. 6459, pp. 59-69. Springer, Heidelberg (2010)

7. Benyon, D., Smyth, M., Helgason, I. (eds.): Presence for Everyone: A Short Guide to Presence Research. The Centre for Interaction Design, Edinburgh Napier University, UK (2009)

8. Peterson, B.: The Influence of Whole-Body Interaction on Wayfinding in Virtual Reality. PhD Thesis, University of Washington (1998)

9. Konami Digital Entertainment, Inc. Dance Dance Revolution (2010), http: / /www. konami.com/ddr/

10. Nintendo, Inc. Wii, http://wii.com/

11. Meehan, M., Whitton, M., Razzaque, S., Zimmon, P., Insko, B., Combe, G., Lok, B., Scheuermann, T., Naik, S., Jerald, J., Harris, M., Antley, A., Brooks, F.: Physiological Reaction and Presence in Stressful Virtual Environments. In: Proc. of ACM SIGGRAPH (2002) 